PROCEEDINGS OF THE

AMERICAN MATHEMATICAL SOCIETY

Volume 138, Number 1, January 2010, Pages 61-73

S 0002-9939(09)10010-2

Article electronically published on August 28, 2009

\title{
CONSECUTIVE CANCELLATIONS IN BETTI NUMBERS OF LOCAL RINGS
}

\author{
MARIA EVELINA ROSSI AND LEILA SHARIFAN
}

(Communicated by Bernd Ulrich)

\begin{abstract}
Let $I$ be a homogeneous ideal in a polynomial ring $P$ over a field. By Macaulay's Theorem there exists a lexicographic ideal $L=\operatorname{Lex}(I)$ with the same Hilbert function as $I$. Peeva has proved that the Betti numbers of $P / I$ can be obtained from the graded Betti numbers of $P / L$ by a suitable sequence of consecutive cancellations. We extend this result to any ideal $I$ in a regular local ring $(R, \mathfrak{n})$ by passing through the associated graded ring. To this purpose it will be necessary to enlarge the list of the allowed cancellations. Taking advantage of Eliahou-Kervaire's construction, we present several applications. This connection between the graded perspective and the local one is a new viewpoint, and we hope it will be useful for studying the numerical invariants of classes of local rings.
\end{abstract}

\section{INTRODUCTION}

For a given ideal $I$ of a regular local ring $(R, \mathfrak{n})$, it is a central problem to get information on the homological invariants of the quotient ring $A=R / I$ under reasonable conditions on the ideal $I$. Often, by means of the Hilbert function of $A$, we try to find information for $A$ being Cohen-Macaulay, Gorenstein or having estimated depth. Deeper information can be deduced from a minimal free resolution of $A$ as an $R$-module. If $I$ is a homogeneous ideal in a polynomial ring $P$ over a field, by Macaulay's Theorem [M] there exists a lexicographic ideal $L=\operatorname{Lex}(I)$ with the same Hilbert function as $I$. A result by Bigatti $\mathrm{Bi}$, Hulett $\mathrm{Hu}$ and Pardue $\left[\mathrm{P}\right.$ says that the graded Betti numbers $\beta_{i j}(P / L)$ are greater than or equal to the corresponding graded Betti numbers $\beta_{i j}(P / I)$. Peeva $[\mathrm{Pe}$, Theorem 1.1] proved that the graded Betti numbers $\beta_{i j}(P / I)$ can be obtained from the graded Betti numbers $\beta_{i j}(P / L)$ by a sequence of zero consecutive cancellations, which are cancellations in the graded Betti numbers of consecutive homological degrees corresponding to the same shift.

The first aim of this paper is to complete Peeva's result in the local case. For any ideal $I$, the Hilbert function of the local ring $A=R / I$ is the Hilbert function of the associated graded ring $g r_{\mathfrak{m}}(A):=\bigoplus_{t>0} \mathfrak{m}^{t} / \mathfrak{m}^{t+1}$, where $\mathfrak{m}=\mathfrak{n} / I$. In particular $g r_{\mathfrak{m}}(A) \simeq P / I^{*}$, where $I^{*}$ is the ideal of the polynomial ring $P=\operatorname{gr}_{\mathfrak{n}}(R)$ generated by the initial forms of the elements of $I$. Then there exists a unique lexicographic

Received by the editors February 11, 2009, and, in revised form, April 17, 2009.

2000 Mathematics Subject Classification. Primary 13D02.

Key words and phrases. Minimal free resolution, filtered module, associated graded module, Betti numbers, lexicographic ideal, standard bases. 
ideal $L=\operatorname{Lex}(I)$ such that $P / L$ has the same Hilbert function as $A$. Starting from a graded free resolution of $g r_{\mathfrak{m}}(A)$ as a $P$-module, we can build up a free resolution of $A$ as an $R$-module which is not necessarily minimal. It will be enough to enlarge the list of the allowed cancellations on the resolution of $L=\operatorname{Lex}(I)$ to obtain a resolution of a local ring $R / I$. The crucial point will be to prove that the Betti numbers of $A$ can be obtained from the graded Betti numbers of $g r_{\mathfrak{m}}(A)$ by a sequence of negative consecutive cancelations (see Theorem 3.1). Most of the results are presented in the more general setting of the filtrations on a module $M$ over a regular local ring $(R, \mathfrak{n})$. This generality enables us to detect the minimal free resolution of the same module through the associated graded modules of suitable good filtrations. For instance, if the ideal $I$ is 2-generated, then $g r_{\mathfrak{m}}(A)$ can be very complicated, but it will be convenient to get information about the free resolution of $I$ (as an $R$-module) by considering the $\mathfrak{n}$-adic filtration on $I$ itself as an $R$-module. In this case the associated graded module $g r_{\mathfrak{n}}(I)=\bigoplus I \mathfrak{n}^{t} / I \mathfrak{n}^{t+1}$ has the same Betti numbers as $I$ (see [HRV]).

Taking advantage of Peeva's result, we can describe in Theorem 4.1 the admissible consecutive cancellations in the minimal free resolution of $L=\operatorname{Lex}(I)$ in order to achieve a minimal free resolution of the local ring $A$. It will be enough to consider the new cancellations coming from Theorem 3.1. Here, we present several applications to find local rings of homogeneous type (Corollary 4.3), to control the depth of $A$ (see Corollary 4.4) and to study the admissible Hilbert functions of special classes of local rings (Corollary 4.5). The fact that the lexicographic ideal $L$ is stable, so its minimal free resolution is given by the well known Eliahou-Kervaire construction, often plays a key role in the above results.

As well as being in the homogeneous context, it should be noted that there are many examples in which the existence of possible consecutive cancellations does not imply the existence of an ideal that those cancellations are realized for it. This is not the case if we consider a perfect ideal of codimension two. Actually, in this situation, we prove that for each sequence of zero or negative consecutive cancellations on the minimal graded free resolution of $L$, we can realize an ideal $I$ in the formal series ring so that $L=\operatorname{Lex}(I)$, including such resolution (see Remark 4.7). Further, we will give a short proof to a well-known characterization of the admissible Hilbert functions of an Artinian Gorenstein local ring of codimension two.

The paper presents several examples, all performed using CoCoA [C].

\section{Preliminaries}

Throughout the paper $(R, \mathfrak{n})$ is a regular local ring with infinite residue field $k$. If $\operatorname{dim} R=n$, then the associated graded ring $g r_{\mathfrak{n}}(R)$ with respect to the $\mathfrak{n}$-adic filtration is the polynomial ring $P=k\left[x_{1}, \ldots, x_{n}\right]$.

Let $M$ be a finitely generated $R$-module. We say, according to the notation in [RV], that a filtration of submodules $\mathbb{M}=\left\{M_{n}\right\}_{n \geq 0}$ on $M$ is an $\mathfrak{n}$-filtration if $\mathfrak{n} M_{n} \subseteq M_{n+1}$ for every $n \geq 0$, and a good (or stable) $\mathfrak{n}$-filtration if $\mathfrak{n} M_{n}=M_{n+1}$ for all sufficiently large $n$. In the following a filtered module $M$ will always be an $R$-module equipped with a good $\mathfrak{n}$-filtration $\mathbb{M}$.

If $\mathbb{M}=\left\{M_{j}\right\}$ is an $\mathfrak{n}$-filtration of $M$, define

$$
g r_{\mathbb{M}}(M)=\bigoplus_{j \geq 0}\left(M_{j} / M_{j+1}\right)
$$


which is a graded $g r_{\mathfrak{n}}(R)$-module in a natural way. It is called the associated graded module to the filtration $\mathbb{M}$, and, for short, it could also be denoted by $M^{*}$. To avoid triviality, we assume that $\operatorname{gr}_{\mathbb{M}}(M)$ is not zero or equivalently $M \neq 0$.

If $m \in M \backslash\{0\}$, we denote by $\nu_{\mathbb{M}}(m)$ the largest integer $p$ such that $m \in M_{p}$ (the so-called valuation of $m$ with respect to $\mathbb{M}$ ), and we denote by $m^{*}$ or $g r_{\mathbb{M}}(m)$ the residue class of $m$ in $M_{p} / M_{p+1}$ where $p=\nu_{\mathbb{M}}(m)$ and call it the initial form of $m$ with respect to $\mathbb{M}$. If $m=0$, we set $\nu_{\mathbb{M}}(m)=+\infty$.

If $N$ is a submodule of $M$, by the Artin-Rees Lemma, the sequence $\left\{N \cap M_{j} \mid j \geq\right.$ $0\}$ is a good $\mathfrak{n}$-filtration of $N$. Since

$$
\left(N \cap M_{j}\right) /\left(N \cap M_{j+1}\right) \simeq\left(N \cap M_{j}+M_{j+1}\right) / M_{j+1},
$$

$g r_{\mathbb{M}}(N)$ is a graded submodule of $g r_{\mathbb{M}}(M)$.

Using (1), it is clear that $g r_{\mathbb{M}}(N)$ is generated by the elements $x^{*}$ with $x \in N$. We write

$$
g r_{\mathbb{M}}(N)=\left\langle x^{*}: x \in N\right\rangle .
$$

On the other hand, it is clear that $\left\{\left(N+M_{j}\right) / N \mid j \geq 0\right\}$ is a good $\mathfrak{n}$-filtration of $M / N$ which we denote by $\mathbb{M} / N$. These graded modules are related by the graded isomorphism

$$
g r_{\mathbb{M} / N}(M / N) \simeq g r_{\mathbb{M}}(M) / g r_{\mathbb{M}}(N) .
$$

For a given filtered module $M$, we recall that an element $g \in M$ is a lifting of an element $h \in g r_{\mathbb{M}}(M)$ if $g r_{\mathbb{M}}(g)=h$.

The morphism of filtered modules $f: M \rightarrow N\left(f\left(M_{p}\right) \subseteq N_{p}\right.$ for every $\left.p\right)$ clearly induces a morphism of graded $g r_{\mathfrak{n}}(R)$-modules

$$
g r(f): g r_{\mathbb{M}}(M) \rightarrow g r_{\mathbb{N}}(N)
$$

Then $g r_{\mathbb{M}}($.$) is a functor from the category of filtered R$-modules into the category of the graded $g r_{\mathfrak{n}}(R)$-modules. Furthermore, we have a canonical embedding $g r_{\mathbb{M}}(\operatorname{Kerf}) \rightarrow \operatorname{Ker}(g r(f))$.

Let $F=\bigoplus_{i=1}^{s} R e_{i}$ be a free $R$-module of rank $s$ and $\nu_{1}, \ldots, \nu_{s}$ be integers. We define the filtration $\mathbb{F}=\left\{F_{p}: p \in \mathbf{Z}\right\}$ on $F$ as follows:

$$
F_{p}:=\bigoplus_{i=1}^{s} \mathfrak{n}^{p-\nu_{i}} e_{i}=\left\{\left(a_{1}, \ldots, a_{s}\right): a_{i} \in \mathfrak{n}^{p-\nu_{i}}\right\} .
$$

From now on, we denote the filtered free $R$-module $F$ by $\bigoplus_{i=1}^{s} R\left(-\nu_{i}\right)$, and we call it a special filtration on $F$. If $(\mathbf{F} ., \delta$.) is a complex of finitely generated free $R$-modules, a special filtration on $\mathbf{F}$. is a special filtration on each $F_{i}$ that makes $(\mathbf{F} ., \delta$.) a complex of filtered modules.

Let $M$ be a finitely generated filtered $R$-module and let $S=\left\{f_{1}, \ldots, f_{s}\right\}$ be a system of elements of $M$ and $\nu_{\mathbb{M}}\left(f_{i}\right)$ be the corresponding valuations. As before let $F=\bigoplus_{i=1}^{s} R e_{i}$ be a free $R$-module of rank $s$ equipped with the filtration $\mathbb{F}$ where $\nu_{i}=\nu_{\mathbb{M}}\left(f_{i}\right)$. Then we denote the filtered free $R$-module $F$ by $\bigoplus_{i=1}^{s} R\left(-\nu_{\mathbb{M}}\left(f_{i}\right)\right)$, hence $\nu_{\mathbb{F}}\left(e_{i}\right)=\nu_{\mathbb{M}}\left(f_{i}\right)$.

Let $\phi: F \rightarrow M$ be a morphism of filtered $R$-modules defined by

$$
\phi\left(e_{i}\right)=f_{i} .
$$

Denote by $\operatorname{Syz}(S)$ the submodule of $F$ generated by the first syzygies of $f_{1}, \ldots, f_{s}$; then $\operatorname{Syz}(S)=\operatorname{Ker} \phi$. 
Definition 2.1. Let $M$ be a filtered module. A subset $S=\left\{f_{1}, \ldots, f_{s}\right\}$ of $M$ is called a standard basis of $M$ if $g r_{\mathbb{M}}(M)=\left\langle f_{1}^{*}, \ldots, f_{s}^{*}\right\rangle$.

By following the initial idea of Robbiano and Valla in [RoV], Shibuta in [Sh] characterized the standard bases of a filtered module as follows:

Theorem 2.2. Let $M$ be a filtered $R$-module, $f_{1}, \ldots, f_{s} \in M$ and $S=\left\{f_{1}, \ldots, f_{s}\right\}$. The following facts are equivalent:

(1) $\left\{f_{1}, \ldots, f_{s}\right\}$ is a standard basis of $M$.

(2) $\left\{f_{1}, \ldots, f_{s}\right\}$ generates $M$, and every element of $\operatorname{Syz}\left(\left\langle f_{1}^{*}, \ldots, f_{s}^{*}\right\rangle\right)$ can be lifted to an element in $\operatorname{Syz}(S)$.

(3) $\left\{f_{1}, \ldots, f_{s}\right\}$ generates $M$ and $\operatorname{Syz}\left(\left\langle f_{1}^{*}, \ldots, f_{s}^{*}\right\rangle\right)=g r_{\mathbb{F}}(\operatorname{Syz}(S))$.

The valuation of an element in a filtered $R$-module often plays the same role as the degree of a homogeneous element in a $P$-module, but we want to point out that there are important differences. For example, in a graded $P$-module the number of the homogeneous generators in each degree is independent from the chosen minimal system; the same property does not hold in the local case with the valuations. For instance, in $A=k[[x, y, z]]$, it is easy to verify that $\left\{x y+z^{3}, x^{2}+\right.$ $\left.x y^{3}, x z^{3}-x y^{4}+y^{2} z^{4}\right\}$ and $\left\{x y+z^{3}, x^{2}+x y^{3}, y^{2} z^{4}\right\}$ are two minimal systems of generators of the same ideal $I$, but they have different valuations with respect to the classical $\mathfrak{m}$-adic filtration, even if they are liftings of minimal generators of $I^{*}=\left(x y, x^{2}, x z^{3}, y^{2} z^{4},-z^{6}, y^{6} z^{3}\right)$, the ideal generated by the initial forms of the elements in $I$.

Using Theorem 2.2, one can prove (see $\mathrm{RSh}$, Theorem 1.8) the following result proved by Robbiano in $\mathrm{R}$.

Theorem 2.3. Let $M$ be a filtered $R$-module and let $(\mathbf{G}$.,d.) be a P-free graded resolution of $\operatorname{gr}_{\mathbb{M}}(M)$ :

$$
\begin{aligned}
\text { G. }: 0 \rightarrow \bigoplus_{i=1}^{\beta_{l}} P\left(-a_{l i}\right) \stackrel{d_{l}}{\rightarrow} \bigoplus_{i=1}^{\beta_{l-1}} P\left(-a_{l-1 i}\right) \\
\stackrel{d_{l-1}}{\rightarrow} \ldots \stackrel{d_{1}}{\rightarrow} \bigoplus_{i=1}^{\beta_{0}} P\left(-a_{0 i}\right) \stackrel{d_{0}}{\rightarrow} g r_{\mathbb{M}}(M) \rightarrow 0 .
\end{aligned}
$$

Then we can build up an $R$-free resolution $(\mathbf{F} ., \delta$.) of $M$ and a special filtration $\mathbb{F}$ on it such that $\operatorname{gr}_{\mathbb{F}}(\mathbf{F})=.\mathbf{G}$..

We recall that $(\mathbf{F} ., \delta$.) is defined by an inductive process. Let us present the inductive steps because the construction will be useful in the following (for more detail we refer the reader to $[\mathrm{RSh})$. Starting from $(\mathbf{G} ., d$.$) , denote by \left\{\epsilon_{0 i}\right\}$ a basis of $G_{0}$. We put $g_{i}=d_{0}\left(\epsilon_{0 i}\right) \in g r_{\mathbb{M}}(M)$ and let $f_{i} \in M$ be such that $g r_{\mathbb{M}}\left(f_{i}\right)=g_{i}$. Then $a_{0 i}=\nu_{\mathbb{M}}\left(f_{i}\right)$. We define the $R$-free module $F_{0}$ of rank $\beta_{0}$ with the induced special filtration $\mathbb{F}_{0}$ on $R^{\beta_{0}}$ :

$$
F_{0}=\bigoplus_{i=1}^{\beta_{0}} R\left(-a_{0 i}\right) .
$$

Denote by $\left\{e_{0 i}\right\}$ a basis of $F_{0}$ and define $\delta_{0}: F_{0} \rightarrow M$ such that $\delta_{0}\left(e_{0 i}\right)=f_{i}$. Since $d_{0}$ is surjective, the $f_{i}$ 's generate a standard basis of $M, \operatorname{Ker}\left(d_{0}\right)=g r_{\mathbb{F}_{0}}\left(\operatorname{Ker}\left(\delta_{0}\right)\right)$ and $F_{0} \stackrel{\delta_{0}}{\rightarrow} M \rightarrow 0$ is exact. We can repeat the same procedure on the successive 
$j$-steps $(j>0)$ of the resolution of $g r_{\mathbb{M}}(M)$. We lift a system of generators of $\operatorname{Ker}\left(d_{j-1}\right)=g r_{\mathbb{F}_{j-1}}\left(\operatorname{Ker}\left(\delta_{j-1}\right)\right)$ to elements in $\operatorname{Ker}\left(\delta_{j-1}\right)$ of valuation $a_{j i}$. Hence we build up the $R$-free modules $F_{j}$ with special filtrations $F_{j}=\bigoplus_{i=1}^{\beta_{j}} R\left(-a_{j i}\right)$ and the differential maps $\delta_{j}: F_{j} \rightarrow F_{j-1}$ such that $g r_{\mathbb{F}_{j}}\left(F_{j}\right)=G_{j}, g r_{\mathbb{F}_{j}}\left(\delta_{j}\right)=d_{j}$. Since, by construction, the lifted elements form a standard basis of $\operatorname{Ker}\left(\delta_{j-1}\right)$, we have

$$
\operatorname{Ker}\left(d_{j}\right)=g r_{\mathbb{F}_{j}}\left(\operatorname{Ker}\left(\delta_{j}\right)\right) \text {, }
$$

and we go on.

It is worth saying that the $R$-free resolution of $M$,

$$
\text { F. : } 0 \rightarrow R^{\beta_{l}} \stackrel{\delta_{l}}{\rightarrow} R^{\beta_{l-1}} \stackrel{\delta_{l-1}}{\rightarrow} \cdots \stackrel{\delta_{1}}{\rightarrow} R^{\beta_{0}} \stackrel{\delta_{0}}{\rightarrow} M \rightarrow 0,
$$

coming from a minimal free resolution of $\operatorname{gr}_{\mathbb{M}}(M)$ is not necessarily minimal. In particular $\left(\mathbf{F} ., \delta\right.$.) is minimal if and only if the Betti numbers of $M$ and $g r_{\mathbb{M}}(M)$ coincide. Our aim is to get information from the numerical invariants of $\mathbf{G}$. in order to achieve a minimal $R$-free resolution of $M$.

Let $N$ be a finitely generated graded module over the polynomial ring $P$. We consider

$$
G_{j}=\bigoplus_{i=1}^{\beta_{j}} P\left(-a_{j i}\right) \stackrel{d_{j}}{\rightarrow} G_{j-1}=\bigoplus_{i=1}^{\beta_{j-1}} P\left(-a_{j-1, i}\right),
$$

a part of a minimal free resolution (G.,d.) of $N$ with $a_{j 1} \leq \cdots \leq a_{j \beta_{j}}$ and $a_{j-1,1} \leq \cdots \leq a_{j-1, \beta_{j-1}}$. Let $1 \leq s \leq \beta_{j}$ and $1 \leq r \leq \beta_{j-1}$ and set

$$
u_{r s}:=a_{j s}-a_{j-1, r}
$$

then the matrix $U_{j}=\left(u_{r s}\right)$ is called the $j$-th degree-matrix of $N$. We say that $U_{j}$ is non-negative if all the entries of $U_{j}$ are non-negative.

We remark that the matrices $U_{j}$ are univocally determined by the graded $P$ module $N$. Denote by $\operatorname{pd}(N)$ the projective dimension of $N$ as a $P$-module.

Proposition 2.4. With the above notation, let $(\mathbf{F} ., \delta$.) be a free resolution of $M$ coming from a graded minimal free resolution $\left(\mathbf{G} ., d\right.$.) of $g r_{\mathbb{M}}(M)$. If the degreematrices $U_{j}$ of $\operatorname{gr}_{\mathbb{M}}(M)$ are non-negative for every $j \leq \operatorname{pd}\left(g r_{\mathbb{M}}(M)\right)$, then $(\mathbf{F} ., \delta$.) is minimal.

Proof. Let $1 \leq j \leq \operatorname{pd}\left(g r_{\mathbb{M}}(M)\right), 1 \leq s \leq \beta_{j}$ and $1 \leq r \leq \beta_{j-1}$, and denote by $\mathcal{M}_{j}^{*}=\left(n_{r s}\right)$ the matrix associated to $d_{j}$ with respect to the bases $\left\{\epsilon_{j 1}, \ldots, \epsilon_{j \beta_{j}}\right\}$ of $G_{j}$ and $\left\{\epsilon_{j-1,1}, \ldots, \epsilon_{j-1, \beta_{j-1}}\right\}$ of $G_{j-1}$. Let $U_{j}=\left(u_{r s}\right)$ be the corresponding degree-matrix. Notice that $\operatorname{deg}\left(n_{r s}\right)=u_{r s}$ if $u_{r s}>0 ; n_{r s}=0$ otherwise.

Following Theorem 2.3 we build up a free resolution $(\mathbf{F} ., \delta$.) of $M$ and denote by $\mathcal{M}_{j}=\left(m_{r s}\right)$ the corresponding matrix associated to $\delta_{j}$. By construction (see (2)) the columns of $\mathcal{M}_{j}$ form a standard basis of $\operatorname{Syz}_{j}(M)$ and the columns of $\mathcal{M}_{j}^{*}$ are the initial forms of the corresponding columns of $\mathcal{M}_{j}$ with respect to the filtration which has been defined on $F_{j-1}$. In particular, the degree matrix $U_{j}$ controls the valuations of entries of $\mathcal{M}_{j}$ with respect to the $\mathfrak{n}$-adic filtration. If $n_{r s} \neq 0$, then $\nu_{\mathfrak{n}}\left(m_{r s}\right)=u_{r s}$; otherwise $\nu_{\mathfrak{n}}\left(m_{r s}\right)>u_{r s}$. So if for all $j$ the entries of the matrix $U_{j}$ are non-negative, then all the entries of $\mathcal{M}_{j}$ belong to $\mathfrak{n}$ and hence $(\mathbf{F} ., \delta$.) is minimal.

The converse of Proposition 2.4 is not true. It is possible that the degree-matrices of $\operatorname{gr}_{\mathbb{M}}(M)$ have negative entries, nevertheless the built up resolution $(\mathbf{F} ., \delta$. $)$ is minimal. This is the case if we consider the local ring $A=k\left[\left[t^{9}, t^{17}, t^{19}, t^{39}\right]\right]$ 
equipped with the $\mathfrak{m}$-adic filtration where $\mathfrak{m}$ is the maximal ideal of $A$ (see $[\mathbf{R S h}$, Example 3.5).

\section{Consecutive Cancellations in Betti numbers of $g r_{\mathbb{M}}(M)$}

The aim of this section is to describe the possible minimal $R$-free resolutions of $M$ obtained from a minimal $P$-free resolution of $g r_{\mathbb{M}}(M)$. We present the following definition which is a suitable adaptation of Peeva's definition.

Given a sequence of numbers $\left\{c_{i}\right\}$ such that $c_{i}=\sum_{j \in \mathbf{N}} c_{i j}$, we obtain a new sequence by a consecutive cancellation as follows: fix an index $i$, and choose $j$ and $j^{\prime}$ such that $j \leq j^{\prime}$ and $c_{i j}, c_{i-1, j^{\prime}}>0$. Then replace $c_{i j}$ by $c_{i j}-1$ and $c_{i-1, j^{\prime}}$ by $c_{i-1, j^{\prime}}-1$ and, accordingly, replace in the sequence $c_{i}$ by $c_{i}-1$ and $c_{i-1}$ by $c_{i-1}-1$. If $j<j^{\prime}$ we call it an $i$-negative consecutive cancellation, and if $j=j^{\prime}$, an $i$-zero consecutive cancellation.

A sequence of consecutive cancellations will mean a finite number of consecutive cancellations performed on a given sequence.

Let $N$ be a homogeneous $P$-module with $P$-free graded resolution given by

$$
\text { G. : } 0 \rightarrow \bigoplus_{i \geq 0} P^{\beta_{l j}}(-j) \stackrel{d_{l}}{\rightarrow} \bigoplus_{i \geq 0} P^{\beta_{l-1, j}}(-j) \stackrel{d_{l-1}}{\rightarrow} \ldots \stackrel{d_{l}}{\rightarrow} \bigoplus_{i \geq 0} P^{\beta_{0 j}}(-j) .
$$

According to the above definition, we will say that the sequence of the Betti numbers $\left\{\beta_{i}=\sum_{j \in \mathbf{N}} \beta_{i j}\right\}$ of $N$ admits an $i$-negative consecutive cancellation (resp. $i$-zero consecutive cancellation) if there exist integers $j<j^{\prime}$ (resp. $j=j^{\prime}$ ) such that $\beta_{i j}, \beta_{i-1, j^{\prime}}>0$.

For example $\cdots \rightarrow P(-3) \oplus P(-5) \oplus P(-6) \rightarrow P^{2}(-2) \oplus P^{2}(-5) \rightarrow \cdots$ admits a zero cancellation $(P(-5), P(-5))$ and a negative cancellation $(P(-3), P(-5))$.

Notice that an $i$-negative consecutive cancellation corresponds to a negative entry of the $i$-th degree-matrix of the graded resolution of $N$. It is clear that a sequence of Betti numbers can admit different diagrams of zero or negative successive cancellations.

Combining Theorem 2.3 and Proposition 2.4, we prove the following result.

Theorem 3.1. Let $(R, \mathfrak{n})$ be a regular local ring and let $M$ be a filtered $R$-module. Then the Betti numbers of $M$ as an $R$-module can be obtained from the Betti numbers of $g r_{\mathbb{M}}(M)$ as a $g r_{\mathfrak{n}}(R)$-module by a sequence of negative consecutive cancellations.

Proof. Let (G., d.) be the minimal free resolution of $\operatorname{gr}_{\mathbb{M}}(M)$ and $\left\{\beta_{i}=\sum_{j \in \mathbf{N}} \beta_{i j}\right\}$ the corresponding sequence of the Betti numbers. By Theorem 2.3, we build up a free resolution $(\mathbf{F} ., \delta$.) of $M$ as an $R$-module from $(\mathbf{G} ., d$.). If $(\mathbf{F} ., \delta$.) is not minimal, then, for some integer $i$, the matrix of the $i$-th differential map $\delta_{i}$

$$
F_{i}=R^{\beta_{i}} \stackrel{\delta_{i}}{\rightarrow} F_{i-1}=R^{\beta_{i-1}}
$$

has an invertible entry. Fix the bases $\left\{e_{i 1}, \ldots, e_{i \beta_{i}}\right\}$ of $F_{i}$ and $\left\{e_{i-1,1}, \ldots, e_{i-1, \beta_{i-1}}\right\}$ of $F_{i-1}$ and let $\mathcal{M}_{i}=\left(m_{r s}\right)$ be the corresponding matrix.

By Proposition 2.4, the $i$-th degree-matrix $U_{i}=\left(u_{r s}\right)$ of $g r_{\mathbb{M}}(M)$ has a negative entry. This means that the sequence $\left\{\beta_{i}=\sum_{j \in \mathbf{N}} \beta_{i j}\right\}$ admits an $i$ negative consecutive cancellation. Let $(p, q)$ be the least couple such that $m_{p q}$ is invertible in $\mathcal{M}_{i}$. Now, to $(\mathbf{F} ., \delta$.), we apply a standard procedure presented for example in 
E. We replace the basis of $F_{i-1}$ by $e_{i-1, j}^{\prime}=e_{i-1, j}$ if $1 \leq j \leq \beta_{i-1}, j \neq p$, and $e_{i-1, p}^{\prime}=\sum_{j=1}^{\beta_{i-1}} m_{j q} e_{i-1, j}$.

It is clear that, with respect to the new basis, the matrices of differential maps in the resolution of $M$ change just for $\delta_{i}$ and $\delta_{i-1}$. Precisely, one can easily check that in $\mathcal{M}_{i}$ for each $r \neq p, m_{r s}$ is replaced by $m_{r s}-m_{p q}^{-1} m_{p s} m_{r q}$ and the entries of the $p$-th row, $m_{p s}$, are replaced by $m_{p q}^{-1} m_{p s}$. So

1) the $q$-th column of $\mathcal{M}_{i}$ is replaced by $(\overbrace{0, \ldots, 0}^{p-1}, 1,0, \ldots, 0)^{t r}$;

2 ) the $p$-th column of $\mathcal{M}_{i-1}$ is replaced by $(0 \cdots 0)^{t r}$.

Therefore, $\delta_{j}\left(e_{i q}\right)=e_{i-1, p}^{\prime}$ and $\delta_{i-1}\left(e_{i-1, p}^{\prime}\right)=0$. Let $H_{j}=0$ if $j \neq i-1, i$ and $H_{i}=\left.F_{i}\right|_{e_{i q}}$ and $H_{i-1}=\left.F_{i-1}\right|_{e_{i-1, p}^{\prime}}$. Thus we have found the following trivial subcomplex of $(\mathbf{F} ., \delta$.):

$$
\text { H. : } \underbrace{0 \rightarrow \cdots \rightarrow 0}_{l-i+1} \rightarrow R \stackrel{i d}{\rightarrow} R \rightarrow \underbrace{0 \rightarrow \cdots \rightarrow 0}_{i},
$$

where $l$ is the length of $(\mathbf{F} ., \delta$. $)$. $\mathbf{H}$. is embedded in $\mathbf{F}$. in such a way that $\widetilde{\mathbf{F}} .=\mathbf{F} . / \mathbf{H}$. is again a free resolution of $M$ which corresponds to cancelling a copy of $R$ in $F_{i}$ and $F_{i-1}$. The matrices of differential maps of $\mathbf{F} . / \mathbf{H}$. are different from those of $(\mathbf{F} ., \delta$.) just for $i-1, i, i+1$. Denote the matrices of the new resolution with respect to the new bases by $\widetilde{\mathcal{M}}_{j}$. Then $\widetilde{\mathcal{M}}_{i-1}$ is obtained by deleting the $p$-th column of $\mathcal{M}_{i-1}, \widetilde{\mathcal{M}}_{i+1}$ is obtained by deleting the $q$-th row of $\mathcal{M}_{i+1}$, and, finally, by deleting the $q$-th column and $p$-th row of $\mathcal{M}_{i}$, we obtain $\widetilde{\mathcal{M}}_{i}$. It is easy to see that the eventually remaining invertible entries of the matrices of the differential maps of the new resolution, $\widetilde{\mathcal{M}}_{j}$, still correspond to the negative entries of the degree matrices of $g r_{\mathbb{M}}(M)$ out of the $p$-th row and $q$-th column of $\mathcal{M}_{i}^{*}$. We can repeat the procedure on $\widetilde{\mathbf{F}}$. until we get a minimal free resolution of $M$.

In general, the Betti numbers of $g r_{\mathfrak{m}}(A)$ can be much greater than those of $A$. For example, if we consider $A=R / I$ with $I=\left(x^{3}-y^{7}, x^{2} y-x t^{3}-z^{6}\right) \subseteq R=k[x, y, z, t]$, then $\beta_{1}(A)=\mu(I)=2$ and $\beta_{1}\left(g r_{\mathfrak{m}}(A)\right)=\mu\left(I^{*}\right)=8$. However, Theorem 3.1 gives a constructive method which relates the minimal free resolution of $A$ and the minimal graded free resolution of $g r_{\mathfrak{m}}(A)$.

We present the following example in order to help the reader better visualize the procedure of Theorem 3.1 .

Example 3.2. Consider $A=k\left[\left[t^{19}, t^{26}, t^{34}, t^{40}\right]\right] \simeq R / I$, where $R=k[[x, y, z, w]]$. Using Theorem 3.1, we want to deduce the Betti numbers of $A$ as an $R$-module from the minimal graded free resolution of $g r_{\mathfrak{m}}(A)=P / I^{*}$ as a $P=k[x, y, z, w]$-module.

In this case we have $\mu(I)=\mu\left(I^{*}\right)=5$. Both $A$ and $g r_{\mathfrak{m}}(A)=P / I^{*}$ are CohenMacaulay; hence they have the same homological dimension. Nevertheless they have different Betti numbers:

$$
0 \rightarrow R \rightarrow R^{5} \rightarrow R^{5} \rightarrow I \rightarrow 0
$$

and

$$
0 \rightarrow P^{2} \rightarrow P^{6} \rightarrow P^{5} \rightarrow I^{*} \rightarrow 0 .
$$

We consider the graded minimal free resolution of $I^{*}$ :

$$
0 \rightarrow P(-5) \oplus P(-8) \rightarrow P^{3}(-4) \oplus P^{2}(-5) \oplus P(-6) \rightarrow P^{5}(-3) \rightarrow I^{*} .
$$


It presents a unique negative cancellation: $(P(-5), P(-6))$. Notice that the Betti numbers of $A$ are obtained from the total Betti numbers of $\operatorname{gr}_{\mathfrak{m}}(A)$ after performing the above cancellation.

It should be noted that there are many examples where the existence of possible consecutive cancellations does not imply the existence of an ideal for which those cancellations are realized.

Example 3.3. Let $I$ be an ideal in the regular local $\operatorname{ring}(R, \mathfrak{n})$ such that $A=R / I$ is Artinian with Hilbert function $\{(1,5,1,1,1)\}$ and $R / \mathfrak{n}$ has characteristic 0 . Elias and Valla (see EV] have proved that the number of the isomorphism classes of the Artinian local rings with this Hilbert function is 5. They have different Betti numbers because they correspond to the different values of the Cohen-Macaulay type $1 \leq \tau \leq 5$. Up to isomorphism, all of them have the same associated graded ring $g r_{\mathfrak{m}}(A)=P / I^{*}$, where

$$
I^{*}=\left(x_{1}^{5}, x_{1} x_{2}, x_{1} x_{3}, x_{1} x_{4}, x_{1} x_{5}, x_{2}^{2}, x_{2} x_{3}, x_{2} x_{4}, x_{2} x_{5}, x_{3}^{2}, x_{3} x_{4}, x_{3} x_{5}, x_{4}^{2}, x_{4} x_{5}, x_{5}^{2}\right)
$$

in $P=k\left[x_{1}, \ldots, x_{5}\right]$. Hence the minimal free resolution of $g r_{\mathfrak{m}}(A)$ is

$$
\begin{array}{r}
\text { G. : } 0 \rightarrow P^{4}(-6) \oplus P(-9) \rightarrow P^{20}(-5) \oplus P^{4}(-8) \rightarrow P^{39}(-4) \oplus P^{6}(-7) \\
\rightarrow P^{36}(-3) \oplus P^{4}(-6) \rightarrow P^{14}(-2) \oplus P(-5) \rightarrow P .
\end{array}
$$

By Theorem 3.1 and by Elias and Valla's result, we know that only 5 diagrams of negative consecutive cancellations can be realized, but the resolution of $g r_{\mathfrak{m}}(A)$ admits a larger number of sequences of negative consecutive cancellations.

The next example shows that we may take advantage of the generality of Theorem 3.1 by using a more advantageous filtration.

Example 3.4. Consider the ideal $I=\left(x^{2} y^{5}, x y z^{6}-z^{9}, y^{5} z^{6}\right)$ in $R=K[[x, y, z]]$. We can prove that $g r_{\mathfrak{m}}(A)=P / I^{*}$, where $I^{*}=\left(x^{2} y^{5}, x y z^{6}, y^{5} z^{6}, y^{4} z^{9}, y^{3} z^{12}, y^{2} z^{15}\right.$, $\left.y z^{18}, z^{21}\right)$ is the ideal of $P=K[x, y, z]$ generated by the initial forms of the elements of $I$. The minimal graded free resolution of $I^{*}$ as a $P$-module is

$$
\begin{gathered}
\text { G. }: 0 \rightarrow P(-15) \oplus P(-17) \oplus P(-19) \oplus P(-21) \rightarrow P(-12) \oplus P(-13) \\
\oplus P^{2}(-14) \oplus P^{2}(-16) \oplus P^{2}(-18) \oplus P^{2}(-20) \oplus P(-22) \rightarrow P(-7) \oplus P(-8) \\
\oplus P(-11) \oplus P(-13) \oplus P(-15) \oplus P(-17) \oplus P(-19) \oplus P(-21),
\end{gathered}
$$

which admits several negative cancellations.

By Theorem [3.1, we can also compute a resolution of $I$ as an $R$-module by considering the $\mathfrak{n}$-adic filtration on $I$ itself. Then we are interested in a resolution of the graded $P$-module $g r_{\mathfrak{n}}(I)=\bigoplus_{n} I \mathfrak{n}^{n} / I \mathfrak{n}^{n+1}$, where $\mathfrak{n}=(x, y, z)$. In [HRV], page 595 , it is proved that

$$
g r_{\mathfrak{n}}(I)=P \oplus P /\left(x^{2} y^{5}\right) \oplus P /\left(x^{2}, x y, x z^{3}, z^{6}\right) .
$$

Hence the minimal free resolution of $g r_{\mathfrak{n}}(I)$ is

$$
\begin{aligned}
\text { G. : } 0 \rightarrow P(-6) & \stackrel{d_{3}}{\rightarrow} P(-3) \oplus P^{2}(-5) \oplus P(-7) \\
& \stackrel{d_{2}}{\rightarrow} P^{2}(-2) \oplus P(-4) \oplus P(-6) \oplus P(-7) \stackrel{d_{1}}{\rightarrow} P^{3},
\end{aligned}
$$

which is easier to handle than those of $g r_{\mathfrak{m}}(A)=P / I^{*}$. 
After the negative consecutive cancellations on $\left(F_{3}, F_{2}, F_{1}\right)$ corresponding to $(P(-6), P(-7), 0),(0, P(-5), P(-7)),(0, P(-5), P(-6)),(0, P(-3), P(-4))$, we get the minimal free resolution of $I$ as

$$
0 \rightarrow R^{2} \rightarrow R^{3} \rightarrow I \rightarrow 0
$$

\section{Consecutive Cancellations in Betti numbers of Lex $(I)$}

Let $I$ be an ideal of the regular local ring $(R, \mathfrak{n})$ and consider the local ring $A=R / I$ with maximal ideal $\mathfrak{m}=\mathfrak{n} / I$. The Hilbert function of $A$ is the Hilbert function of $g r_{\mathfrak{m}}(A)=P / I^{*}$, where $I^{*}$ is the ideal of the polynomial ring $P$ generated by the initial forms of the elements of $I$. We denote by $L=\operatorname{Lex}(I)$ the (unique) lexicographic ideal of $P$ such that $P / L$ has the same Hilbert function as $g r_{\mathfrak{m}}(A)$. While the resolution of $g r_{\mathfrak{m}}(A)$ is in general unknown, the resolution of $P / \operatorname{Lex}(I)$ can be easily determined.

Combining Peeva's result and Theorem 3.1, we immediately get the following theorem.

Theorem 4.1. Let I be an ideal of the regular local ring $(R, \mathfrak{n})$. The Betti numbers of $R / I$ can be obtained from the Betti numbers of $P / \operatorname{Lex}(I)$ by a sequence of negative and zero consecutive cancellations.

In order to apply Theorem 4.1, we are interested in finding the zero and negative consecutive cancellations in $\left\{\beta_{i}(P / L)\right\}$. For a monomial $m$, we set $\max (m)=$ $\max \left\{p \mid x_{p}\right.$ divides $\left.m\right\}$. We can easily get an extension of Proposition 1.2 in [Pe] for also testing the existence of negative cancellations.

Proposition 4.2. Let $L$ be a lexicographic ideal of $P$. If the sequence of the Betti numbers of $L$ admits an $i$-zero or $i$-negative consecutive cancellation, then the following two conditions are satisfied:

(1) L has a minimal monomial generator $m$ with $\max (m) \geq i-1$.

(2) L has a minimal monomial generator $m^{\prime}$ with $\max \left(m^{\prime}\right) \geq i$ and $\operatorname{deg}\left(m^{\prime}\right)<$ $\operatorname{deg}(m)$.

Proof. Let $G(L)$ be the set of (unique) minimal monomial generators of $L$. The minimal graded free resolution of $P / L$ is provided by a construction of Eliahou and Kervaire [EK]. The resolution has basis

$$
\left\{e_{\left(m ; j_{1}, \ldots, j_{i-1}\right)} \mid m \in G(L), 1 \leq j_{1}<\cdots<j_{i-1}<\max (m)\right\}
$$

in homological degree $i$ and $e_{\left(m ; j_{1}, \ldots, j_{i-1}\right)}$ has degree $i-1+\operatorname{deg}(m)$. Thus, the first condition is equivalent to $\beta_{i-1, i-2+\operatorname{deg}(m)} \neq 0$ for some monomial $m$ in $G(L)$, and the second condition is equivalent to $\beta_{i, i-1+\operatorname{deg}\left(m^{\prime}\right)} \neq 0$ for some monomial $m^{\prime}$ in $G(L)$ such that $\operatorname{deg}\left(m^{\prime}\right)<\operatorname{deg}(m)$. Both conditions together are equivalent to the fact that the degree matrix $U_{i}$ of $P / L$ has a negative or zero entry.

The next corollaries are easy applications of Theorem 4.1.

Corollary 4.3. Let $I$ be an ideal in the regular local ring $(R, \mathfrak{n})$. If $L=\operatorname{Lex}(I)$ is minimally generated in two successive degrees, then $\beta_{i}(R / I)=\beta_{i}\left(P / I^{*}\right)$ for each $i \geq 0$. 
Proof. Assume that $L$ is generated in degrees $a$ and $a+1$. By the proof of Proposition 4.2 the minimal free resolution of $P / L$ has the following shape:

$$
\begin{aligned}
0 & \rightarrow P^{\beta_{h, a+h-1}}(-a-h+1) \oplus P^{\beta_{h, a+h}}(-a-h) \\
& \rightarrow \cdots \rightarrow P^{\beta_{2, a+1}}(-a-1) \oplus P^{\beta_{2, a+2}}(-a-2) \\
& \rightarrow P^{\beta_{1, a}}(-a) \oplus P^{\beta_{1, a+1}}(-a-1) \rightarrow P \rightarrow 0 .
\end{aligned}
$$

Therefore the only possible cancellations in the Betti numbers of $P / L$ are zero consecutive cancellations; hence by Theorem 3.1 the conclusion follows.

In the following, $\mu(\mathrm{)})$ will denote the minimal number of generators. The next corollary extends to the local case a recent result by Hibi and Murai in [HM].

Corollary 4.4. Let $I \subseteq \mathfrak{n}^{2}$ be a non-zero ideal of the regular local ring $(R, \mathfrak{n})$ of dimension $n$. Assume $\mu(\operatorname{Lex}(I)) \leq n$; then

(1) $\operatorname{dim}(R / I)=n-1$.

(2) $\operatorname{depth}(R / I)=\operatorname{depth}\left(P / I^{*}\right)=\operatorname{depth}(P / \operatorname{Lex}(I))=n-\mu(\operatorname{Lex}(I))$.

(3) $\mu(\operatorname{Lex}(I))=p d(R / I)$.

(4) $\beta_{h}(R / I)=\beta_{h}\left(P / I^{*}\right)=\beta_{h}(P / \operatorname{Lex}(I))=1$, where $h=p d(R / I)$.

Proof. Let $G=\left\{m_{1}, m_{2}, \ldots, m_{h}\right\}$ be the (unique) minimal monomial generating set for the monomial ideal $\operatorname{Lex}(I)$ in $P=k\left[x_{1}, \ldots, x_{n}\right]$, where $1<\operatorname{deg}\left(m_{1}\right) \leq$ $\operatorname{deg}\left(m_{2}\right) \leq \cdots \leq \operatorname{deg}\left(m_{h}\right)$ and where $m_{i+1}<$ Lex $m_{i}$ if $\operatorname{deg}\left(m_{i}\right)=\operatorname{deg}\left(m_{i+1}\right)$. By considering $x_{1}>x_{2}>\cdots$ Hibi and Murai in Proposition 1.2 of [HM] have showed that for every $k \leq n$ we have $m_{k}=x_{1}^{s_{1}} x_{2}^{s_{2}} \cdots x_{k}^{s_{k}+1}$, where $s_{1}=\operatorname{deg} m_{1}-1 \geq 1$ and $s_{i}=\operatorname{deg} m_{i}-\operatorname{deg} m_{i-1}$ for $i=2,3, \ldots, h$. So clearly $\operatorname{Lex}(I) \subseteq\left(x_{1}\right)$ and hence $\operatorname{dim}(P / \operatorname{Lex}(I))=n-1$. By the Eliahou-Kervaire resolution [EK], the tail of the minimal free resolution of $P / \operatorname{Lex}(I)$ is

(4) $0 \rightarrow P\left(-h-\operatorname{deg}\left(m_{h}\right)+1\right) \rightarrow P^{h-1}\left(-h-\operatorname{deg}\left(m_{h}\right)+2\right) \oplus P\left(-h-\operatorname{deg}\left(m_{h-1}\right)+2\right)$.

The conclusion now follows by Theorem 3.1 and Theorem 4.1 .

The following result demonstrates an application in studying the admissible Hilbert functions of particular classes of local rings.

Corollary 4.5. Let $\left\{\left(1, n, h_{2}, \ldots, h_{t}, 1, \ldots, 1,0,0, \ldots\right)\right\}$ be the Hilbert function of an Artinian Gorenstein local ring $A=R / I$. If $t \geq n$, then $h_{t} \leq n$.

Proof. Suppose that $h_{t}>n$. Let $G=\left\{m_{1}, \ldots, m_{h}\right\}$ be the (unique) minimal monomial generating set of the monomial ideal $\operatorname{Lex}(I)$, where $\operatorname{deg}\left(m_{1}\right) \leq \operatorname{deg}\left(m_{2}\right) \leq$ $\cdots \leq \operatorname{deg}\left(m_{h}\right)$ and where $m_{i+1}<_{\text {Lex }} m_{i}$ if $\operatorname{deg}\left(m_{i}\right)=\operatorname{deg}\left(m_{i+1}\right)$. One can check that $m_{h}=x_{n}^{s+1}$, where $s$ is the degree of the $h$-vector, and for $1 \leq i \leq n, m_{h-i}=$ $x_{n-1}^{i} x_{n}^{t-i+1}$. Again by the Eliahou-Kervaire resolution, the tail of the minimal free resolution of $P / \operatorname{Lex}(I)$ is

$$
\begin{aligned}
0 & \rightarrow \bigoplus_{j<n+t} P^{\beta_{n, j}}(-j) \oplus P^{\beta n, n+t}(-n-t) \oplus P(-n-s) \\
& \rightarrow \bigoplus_{j<n+t-1} P^{\beta_{n-1, j}}(-j) \oplus P^{\beta n-1, n+t-1}(-n-t+1) \oplus P^{n-1}(-n-s+1),
\end{aligned}
$$

where $\beta_{n, n+t} \geq n$. By Theorem 4.1 we get $\beta_{n}(R / I)>1$, which is a contradiction. 
For instance, the above result says that $\{(1,3,4,4,1,1,1)\}$ cannot be the Hilbert function of any Artinian Gorenstein local ring $A=K[[x, y, z]] / I$. In fact, $L=$ $\operatorname{Lex}(I)$ should be the ideal $L=\left(x^{2}, x y, x^{2} z, x z^{2}, x y z, y^{4}, y^{3} z, y^{2} z^{2}, y z^{3}, z^{7}\right)$. The minimal free resolution of $P / L$ is

$$
\begin{aligned}
\mathbf{G}: 0 & \rightarrow P(-5) \oplus P^{3}(-6) \oplus P(-9) \rightarrow P(-3) \oplus P^{2}(-4) \oplus P^{7}(-5) \oplus P^{2}(-8) \\
& \rightarrow P^{2}(-2) \oplus P(-3) \oplus P^{4}(-4) \oplus P(-7) \rightarrow P .
\end{aligned}
$$

However, if we consider any sequence of zero and negative consecutive cancellations, we get $\beta_{3}(A) \geq 2$, which contradicts the assumption that $A$ is Gorenstein.

We now present an investigation in codimension two. Assume $I$ is an ideal of a regular local ring $(R, \mathfrak{n})$ of dimension two such that $A=R / I$ is Artinian.

By Macaulay's Theorem, for some integer $d$ one has that the Hilbert function of $A$ is

$$
\left\{\left(1,2, \ldots, d, h_{d}, \ldots, h_{s}, 0,0, \ldots\right)\right\}
$$

where $s$ is the socle degree and $d=h_{d-1} \geq h_{d} \geq h_{d+1} \geq \cdots \geq h_{s} \geq 1$. We consider the corresponding lexicographic ideal $L$ in $P=k[x, y]$. It can be written as

$$
L=\left(x^{d}, x^{d-1} y^{k_{1}}, \ldots, y^{k_{d}}\right)
$$

with $0=k_{0}<k_{1}<\cdots<k_{d}$. For every $j>0$ define

$$
e_{j}:=\left|h_{j}-h_{j-1}\right| \text {. }
$$

We consider $h_{j}=j+1$ if $j \leq d$ and $h_{j}=0$ if $j>s$. It is easy to see that the minimal number of generators of degree $d$ of $L$ is $e_{d}+1$ and, for $j>d$, the minimal number of generators of degree $j$ of $L$ is $e_{j}$. Notice that $\sum_{j>d} e_{j}=d$. The minimal free resolution of $P / L$ is given by $0 \rightarrow F_{2} \rightarrow F_{1} \rightarrow P \rightarrow P / L \rightarrow 0$, where $F_{2}$ and $F_{1}$ have rank $d$ and $d+1$ respectively. In particular,

$$
F_{2}=\bigoplus_{j \geq 0} P^{e_{d+j}}(-d-j-1) \text { and } F_{1}=P^{e_{d}+1}(-d) \bigoplus_{j \geq 1} P^{e_{d+j}}(-d-j) .
$$

As a consequence of Theorem 4.1 we can present an easy proof of a result stated by Macaulay, later proved by Iarrobino in [I] and by Bertella in [B] with different methods and technical devices.

Corollary 4.6. Let $\left\{\left(1,2, \ldots, d, h_{d}, \ldots, h_{s}, 0,0, \ldots\right)\right\}$ be the Hilbert function of an Artinian Gorenstein local ring $A=R / I$; then $e_{j} \leq 1$ for every $j>0$.

Proof. Let $L$ be the lexicographic ideal of $I$. With the above notation, we have

$$
F_{2}=\bigoplus_{j \geq 0} P^{e_{d+j}}(-d-j-1) \text { and } F_{1}=P^{e_{d}+1}(-d) \bigoplus_{j \geq 1} P^{e_{d+j}}(-d-j)
$$

with $e_{s+1}=1\left(h_{s}=1\right)$ and $e_{i}=0$ for $i \geq s+2$. Since $R / I$ is Gorenstein, $\beta_{2}(R / I)=$ 1 and, by Theorem 4.1, the minimal free resolution of $R / I\left(0 \rightarrow R \rightarrow R^{2} \rightarrow R\right)$ is obtained from the resolution of $L$ by a sequence of zero and negative cancellations. Now $F_{2}=P(-s-2) \oplus\left[\bigoplus_{d \leq i \leq s} P^{e_{i}}(-i-1)\right]$. Since $P(-s-2)$ cannot be cancelled, we should cancel in $F_{2}$ the remaining $\left[\bigoplus_{d \leq i \leq s} P^{e_{i}}(-i-1)\right]$. Let $t \leq s$ be the biggest integer such that $e_{t} \neq 0$. The only chance to cancel $P^{e_{t}}(-(t+1))$ in $F_{2}$ is through $P(-s-1)$ in $F_{1}$; hence $e_{t}=1$. Continuing this procedure $d-1$ times, we conclude that $e_{i} \leq 1$ for every $i \geq 1$. 
Following essentially the same idea as in [B], we can realize an ideal $I$ in $k[[x, y]]$ coming from any sequence of zero and negative cancellations in the resolution of $L=\operatorname{Lex}(I)$.

Remark 4.7. Let $0 \rightarrow F_{2} \rightarrow F_{1} \rightarrow L \rightarrow 0$ be a minimal $P$-free resolution of

$$
L=\left(x^{d}, x^{d-1} y^{k_{1}}, \ldots, y^{k_{d}}\right)
$$

with $0=k_{0}<k_{1}<\cdots<k_{d}$. The matrix $M$ of the differential map $F_{2} \rightarrow F_{1}$ is the well-known Hilbert-Burch matrix whose columns can be described as follows:

$$
\left(\sigma_{1}^{t r}, \ldots, \sigma_{d}^{t r}\right), \quad \text { where } \quad \sigma_{i}=(\overbrace{0, \ldots, 0}^{i-1}, y^{k_{i}-k_{i-1}},-x, 0, \ldots, 0) \quad i=1, \ldots, d .
$$

It is clear that each cancellation in the resolution of $L$ corresponds to an element in position $(i, j)$ in $M$ with non-positive value in the associated degree-matrix. Consider the sequence of cancellations corresponding to the elements in position $\left\{\left(l_{1}, t_{1}\right), \ldots,\left(l_{k}, t_{k}\right)\right\}$ and let $I$ be the ideal of $R=k[[x, y]]$ generated by the maximal minors of the following matrix:

$$
M^{\prime}:=M+\left(\alpha_{i j}\right)_{\substack{i=1, \ldots, d+1 \\ j=1, \ldots, d}},
$$

where

$$
\alpha_{i j}= \begin{cases}1, & \text { if }(i, j) \in\left\{\left(l_{1}, t_{1}\right), \ldots,\left(l_{k}, t_{k}\right)\right\} \\ 0, & \text { otherwise }\end{cases}
$$

We can prove that $L=\operatorname{Lex}(I)$ and the corresponding ideal $I^{*}$ follows by the above construction by performing the only zero cancellations. This construction holds in the general case of an Artinian local ring of codimension two, not necessarily Gorenstein, by using the same procedure as in [B], Theorem 2.4.

The next example realizes the above construction.

Example 4.8. Let $H=\{(1,2,3,4,3,3,3,2,2,1)\}$; then $L=\left\langle x^{4}, x^{3} y, x^{2} y^{5}, x y^{8}, y^{10}\right\rangle$. The minimal free resolution of $P / L$ is

$0 \rightarrow P(-5) \oplus P(-8) \oplus P(-10) \oplus P(-11) \rightarrow P^{2}(-4) \oplus P(-7) \oplus P(-9) \oplus P(-10) \rightarrow P$.

The resolution admits two negative cancellations: $(P(-5), P(-7)),(P(-8), P(-9))$ and the zero cancellation $(P(-10), P(-10))$. According to Remark 4.7 the sequence obtained by the zero and negative cancellations $0 \rightarrow R \rightarrow R^{2} \rightarrow R$ can be realized by the ideal $I$ generated by the maximal minors of the following matrix:

$$
\left(\begin{array}{cccc}
y & 0 & 0 & 0 \\
-x & y^{4} & 0 & 0 \\
1 & -x & y^{3} & 0 \\
0 & 1 & -x & y^{2} \\
0 & 0 & 1 & -x
\end{array}\right)
$$

We have $I=\left\langle x^{4}-x^{2} y^{2}-x^{2} y^{3}-x^{2} y^{4}+y^{6}, x^{3} y-x y^{3}-x y^{4}\right\rangle$ and $L=\operatorname{Lex}(I)$. Furthermore, the minimal free resolution of $P / I^{*}$ is obtained by performing the only zero cancellation:

$$
0 \rightarrow P(-5) \oplus P(-8) \oplus P(-11) \rightarrow P^{2}(-4) \oplus P(-7) \oplus P(-9) \rightarrow P
$$


and $I^{*}=\left\langle x^{4}-x^{2} y^{2}, x^{3} y-x y^{3}, x^{2} y^{5}-y^{7}, x y^{8}\right\rangle$ is given by the maximal minors of the matrix

$$
\left(\begin{array}{cccc}
y & 0 & 0 & 0 \\
-x & y^{4} & 0 & 0 \\
0 & -x & y^{3} & 0 \\
0 & 0 & -x & y^{2} \\
0 & 0 & 1 & -x
\end{array}\right)
$$

\section{REFERENCES}

[B] V. Bertella, Hilbert function of local Artinian level rings in codimension two, J. Algebra 321 (2009), no. 5, 1429-1442.

[Bi] A.M. Bigatti, Upper bounds for the Betti numbers of a given Hilbert function, Comm. Algebra 21 (7) (1993), 2317-2334. MR1218500 (94c:13014)

[C] CoCoA Team, CoCoA: a system for doing Computations in Commutative Algebra, available at http://cocoa.dima.unige.it.

[E] D. Eisenbud, The Geometry of Syzygies, A Second Course in Commutative Algebra and Algebraic Geometry, Graduate Texts in Mathematics, 229, Springer-Verlag, New York, 2005. MR2103875 (2005h:13021)

[EK] S. Eliahou, M. Kervaire, Minimal resolutions of some monomial ideals, J. Algebra 129 (1990), no. 1, 1-25. MR1037391 (91b:13019)

[EV] J. Elias, G. Valla, Structure theorems for certain Gorenstein ideals, Michigan Math. J. 57 (2008), 269-292. MR 2492453

[HM] T. Hibi, S. Murai, The depth of an ideal with a given Hilbert function, Proc. Amer. Math. Soc. 136 (2008), no. 5, 1533-1538. MR.2373580 (2009b:13028)

[HRV] J. Herzog, M. E. Rossi, G. Valla, On the depth of the symmetric algebra, Trans. Amer. Math. Soc. 296 (1986), no. 2, 577-606. MR846598 (87f:13009)

$[\mathrm{Hu}]$ H. A. Hulett, Maximum Betti numbers of homogeneous ideals with a given Hilbert function, Comm. Algebra 21 (1993), no. 7, 2335-2350. MR.1218501 (94c:13015)

[I] A. Iarrobino, Punctual Hilbert schemes, Mem. Amer. Math. Soc. 10, no. 188 (1977). MR 0485867(58:5667)

[M] F. Macaulay, Some properties of enumeration in the theory of modular systems, Proc. London Math. Soc. 26 (1927), 531-555.

[P] K. Pardue, Deformation classes of graded modules and maximal Betti numbers, Illinois J. Math. 40 (1996), no. 4, 564-585. MR 1415019 (97g:13029)

[Pe] I. Peeva, Consecutive cancellations in Betti numbers, Proc. Amer. Math. Soc. 132 (2004), no. 12, 3503-3507. MR2084070 (2006a:13023)

[R] L. Robbiano, Coni tangenti a singolaritá razionali, Curve algebriche, Istituto di Analisi Globale, Firenze, 1981.

[RoV] L. Robbiano, G. Valla, On the equations defining tangent cones, Math. Proc. Cambridge Philos. Soc. 88 (1980), no. 2, 281-297. MR578272 (81i:14004)

[RSh] M. E. Rossi, L. Sharifan, Extremal Betti numbers of filtered modules over a regular local ring, to appear in J. of Algebra, in press.

[RV] M. E. Rossi, G. Valla, Hilbert function of filtered modules, arXiv:0710.2346v1 [math.AC].

[Sh] T. Shibuta, Cohen-Macaulayness of almost complete intersection tangent cones, J. Algebra 319 (8) (2008), 3222-3243. MR2408315 (2009b:13061)

Department of Mathematics, University of Genoa, Via Dodecaneso 35, 16146 Genoa, ITALY

E-mail address: rossim@dima.unige.it

Faculty of Mathematics and Computer Science, Amirkabir University of Technology, 424, Hafez Avenue, 15914 Tehran, Iran

E-mail address: leila-sharifan@aut.ac.ir 